\title{
Weak mixing angle in the Thomson limit
}

\section{Jens Erler and Rodolfo Ferro-Hernández}

Departamento de Física Teórica, Instituto de Física, Universidad Nacional Autónoma de México, Circuito de la Investigación Científica s/n, Ciudad Universitaria, Coyoacán, CDMX, México

E-mail: erler@fisica.unam.mx, ferrohr@estudiantes.fisica.unam.mx

ABSTRACT: We present a calculation of the weak mixing angle in the $\overline{\mathrm{MS}}$ renormalization scheme which is relevant for experiments performed at very low energies or momentum transfers. We include higher orders in the perturbative QCD expansion, as well as updated phenomenological and theoretical input, and obtain the result $\sin ^{2} \hat{\theta}_{W}(0)=0.23868(5)(2)$ for the reference values $\hat{\alpha}_{s}\left(M_{Z}\right)=0.1182$ and $\hat{m}_{c}\left(\hat{m}_{c}\right)=1.272 \mathrm{GeV}$. The first quoted error is from the current Standard Model evaluation of the mixing angle at the $Z$ boson mass scale. The second error represents the theoretical and parametric uncertainties induced by the evolution to the Thomson limit and is discussed in detail.

Keywords: Quark Masses and SM Parameters, Resummation

ARXIV EPRINT: 1712.09146 


\section{Contents}

1 Introduction 1

2 Renormalization group evolution 3

2.1 Matching conditions 5

$\begin{array}{llr}3 & \text { Implementation of experimental input } & 6\end{array}$

4 Singlet contribution $\quad 8$

5 Flavor separation $\quad 10$

5.1 Experimental data 11

$\begin{array}{ll}5.2 \text { Lattice data } & 12\end{array}$

$\begin{array}{lll}5.3 & \text { Threshold masses } & 13\end{array}$

$\begin{array}{lll}\text { 5.3.1 Heavy quarks } & 13\end{array}$

$\begin{array}{lll}\text { 5.3.2 Light quarks } & 14\end{array}$

6 Theoretical uncertainties $\quad 15$

$\begin{array}{llr}7 & \text { Results and conclusions } & 16\end{array}$

$\begin{array}{lr}\text { A Calculations of } \alpha\left(M_{Z}^{2}\right) & 18\end{array}$

$\begin{array}{ll}\text { B Calculation of } \Delta_{\text {disc }} \hat{\alpha} & 19\end{array}$

\section{Introduction}

The electroweak sector of the Standard Model is based on the gauge symmetry group $S U(2)_{L} \times U(1)_{Y}$. The weak mixing angle, $\theta_{W}$, is a parameter that describes the mixing of the gauge bosons related to the $U(1)_{Y}$ and the third component of $S U(2)_{L}$ to give rise to the mass eigenstates of the photon and the $Z$ boson. In terms of the couplings $g$ of $S U(2)_{L}$ and $g^{\prime}$ of $\mathrm{U}(1)_{Y}$ one has

$$
\hat{s}^{2} \equiv \sin ^{2} \hat{\theta}_{W}=\frac{g^{\prime 2}}{g^{2}+g^{\prime 2}} .
$$

Since it is given explicitly by gauge couplings, $\sin ^{2} \hat{\theta}_{W}$ depends on the energy scale and is governed by a renormalization group equation (RGE) $[1,2]$.

One of the tests of the Standard Model is to evolve the weak mixing angle from high to low energies and compare it with experimental extractions at lower squared momentum transfers, $Q^{2}$. For example, the Qweak experiment [3] at Jefferson Laboratory (JLab) has 
measured the weak charge of the proton, $Q_{W}(p) \sim 1-4 \sin ^{2} \theta_{W}$, in polarized electron scattering from a fixed liquid hydrogen target at $Q^{2} \approx 0.026 \mathrm{GeV}^{2}$. The same observable, but at an even lower $Q^{2} \approx 0.0045 \mathrm{GeV}^{2}$, will also be targeted by the P2 experiment [4] at the MESA facility which is currently under construction at the University of Mainz in Germany. In a very similar setup, the MOLLER Collaboration [5] at JLab will build and improve on the completed E158 experiment [6] at SLAC (that occurred at almost the same $Q^{2}$ as Qweak) and measure the analogous weak charge of the electron, $Q_{W}(e)$, in polarized Møller scattering at $Q^{2} \approx 0.0056 \mathrm{GeV}^{2}$. The PVDIS Collaboration [7] at the $6 \mathrm{GeV}$ CEBAF complex at JLab scattered polarized electrons deep-inelastically from deuterium, and the SoLID Collaboration [8] will increase the PVDIS precision in the future by benefiting from the energy upgraded CEBAF and a correspondingly higher and broader $Q^{2}$ range. Other approaches include neutrino and anti-neutrino deep inelastic scattering [9], $\bar{\nu}-e$ scattering near nuclear reactors [10], and parity violation in atoms [11] and ions [12]. For more details, see the recent reviews on low energy measurements of the weak mixing angle [13], on the weak neutral current [14], and on weak polarized electron scattering [15].

Since QCD at low energies does not allow for reliable perturbative calculations, the theoretical uncertainty of the RGE running from the $Z$-pole to low energies arises dominantly from the hadronic region. A phenomenological approach to address this region was developed in ref. [2]. Working in the $\overline{\mathrm{MS}}$ scheme, ${ }^{1}$ the main idea was to relate the case of the weak mixing angle to that of the electromagnetic coupling, $\hat{\alpha}$, as far as possible, and then to consider both maximal and minimal SU(3) flavor symmetry breaking to constrain the flavor separation of the three light quarks $(u, d, s)$. In the present work, we extend the analysis to the next order in the strong coupling constant, $\hat{\alpha}_{s}$, and introduce a number of new elements. We employ the most recent values and uncertainties of the input parameters, such as $\hat{\alpha}_{s}$ and the heavy quark masses. The hadronic vacuum polarization contribution to the RGE running of $\hat{\alpha}$ is obtained dispersively from $e^{+} e^{-}$annihilation data for hadronic final states, which are supplemented by isospin rotated $\tau$ decay spectral functions corrected for isospin breaking effects $[16,17]$. We tie experimental data [16] and lattice gauge theory calculations $[18,19]$ together to obtain the individual contributions of strange and first generation quarks. This flavor separation at the quark level to high accuracy is consistent with and almost an order of magnitude more precise than previous calculations $[1,2]$. It is also necessary to constrain OZI-rule [20-22] violating effects, for which we utilize the recent lattice gauge theory calculation of disconnected contributions to the anomalous magnetic moment of the muon [23]. These refinements allow for significant reduction of the theoretical uncertainty of the RGE evolution. As a by-product, our method sheds light on the dual description of quarks and hadrons in the non-perturbative regime and may open new ways to extract the strange quark mass from the electro-production of hadrons.

The paper is organized as follows: section 2 presents the RGE of the weak mixing angle up to five loop accuracy, and the matching conditions for $\hat{\alpha}$ and $\sin ^{2} \theta_{W}$. In section 3 we perform the conversion of the hadronic vacuum polarization contribution to the running of $\hat{\alpha}$ from the on-shell scheme, where it is most directly obtained, to the $\overline{\mathrm{MS}}$ scheme (appendix A

\footnotetext{
${ }^{1}$ Quantities defined in the $\overline{\mathrm{MS}}$ scheme will be denoted by a caret.
} 


\begin{tabular}{|lrlr|}
\hline boson & $\gamma_{i}$ & fermion & $\gamma_{i}$ \\
\hline real scalar & 1 & chiral fermion & 4 \\
complex scalar & 2 & Majorana fermion & 4 \\
massless gauge boson & -22 & Dirac fermion & 8 \\
\hline
\end{tabular}

Table 1. RGE contributions of different particle types, where the minus sign is indicative for the asymptotic freedom in non-Abelian gauge theories.

contains a brief discussion of various calculations of $\alpha\left(M_{Z}\right)$ ). Section 4 describes the calculation of the singlet contribution to the weak mixing angle, with some details given in appendix B. In section 5 the flavor separation (contributions of light and strange quarks) is addressed and threshold masses are calculated. In section 6 theoretical uncertainties are discussed in detail, and section 7 offers our final results and conclusions.

\section{Renormalization group evolution}

In an approximation in which all fermions are either massless and active or infinitely heavy and decoupled, the RGE for the electromagnetic coupling in the $\overline{\mathrm{MS}}$ scheme [24], $\hat{\alpha}$, can be written in the form [2],

$$
\mu^{2} \frac{d \hat{\alpha}}{d \mu^{2}}=\frac{\hat{\alpha}^{2}}{\pi}\left[\frac{1}{24} \sum_{i} K_{i} \gamma_{i} Q_{i}^{2}+\sigma\left(\sum_{q} Q_{q}\right)^{2}\right],
$$

where the sum is over all active particles in the relevant energy range. The $Q_{i}$ are the electric charges, while the $\gamma_{i}$ are constants depending on the field type and shown in table 1 . The $K_{i}$ and $\sigma$ contain higher-order corrections and are given by [25],

$$
\begin{aligned}
K_{i}=N_{i}^{c}\left\{1+\frac{3}{4} Q_{i}^{2} \frac{\hat{\alpha}}{\pi}+\frac{\hat{\alpha}_{s}}{\pi}+\frac{\hat{\alpha}_{s}^{2}}{\pi^{2}}\left[\frac{125}{48}-\frac{11}{72} n_{q}\right]\right. \\
+\frac{\hat{\alpha}_{s}^{3}}{\pi^{3}}\left[\frac{10487}{1728}+\frac{55}{18} \zeta_{3}-n_{q}\left(\frac{707}{864}+\frac{55}{54} \zeta_{3}\right)-\frac{77}{3888} n_{q}^{2}\right] \\
+\frac{\hat{\alpha}_{s}^{4}}{4 \pi^{4}}\left[\frac{2665349}{41472}+\frac{182335}{864} \zeta_{3}-\frac{605}{16} \zeta_{4}-\frac{31375}{288} \zeta_{5}\right. \\
-n_{q}\left(\frac{11785}{648}+\frac{58625}{864} \zeta_{3}-\frac{715}{48} \zeta_{4}-\frac{13325}{432} \zeta_{5}\right) \\
\left.\left.-n_{q}^{2}\left(\frac{4729}{31104}-\frac{3163}{1296} \zeta_{3}+\frac{55}{72} \zeta_{4}\right)+n_{q}^{3}\left(\frac{107}{15552}+\frac{1}{108} \zeta_{3}\right)\right]\right\},
\end{aligned}
$$

and,

$$
\begin{aligned}
\sigma=\frac{\hat{\alpha}_{s}^{3}}{\pi^{3}}\left[\frac{55}{216}-\frac{5}{9} \zeta_{3}\right]+\frac{\hat{\alpha}_{s}^{4}}{\pi^{4}} & {\left[\frac{11065}{3456}-\frac{34775}{3456} \zeta_{3}+\frac{55}{32} \zeta_{4}+\frac{3875}{864} \zeta_{5}\right.} \\
& \left.-n_{q}\left(\frac{275}{1728}-\frac{205}{576} \zeta_{3}+\frac{5}{48} \zeta_{4}+\frac{25}{144} \zeta_{5}\right)\right],
\end{aligned}
$$




\begin{tabular}{|ccccc|}
\hline Energy range & $\lambda_{1}$ & $\lambda_{2}$ & $\lambda_{3}$ & $\lambda_{4}$ \\
\hline $\bar{m}_{t} \leq \mu$ & $\frac{9}{20}$ & $\frac{289}{80}$ & $\frac{14}{55}$ & $\frac{9}{20}$ \\
$M_{W} \leq \mu<\bar{m}_{t}$ & $\frac{21}{44}$ & $\frac{625}{176}$ & $\frac{6}{11}$ & $\frac{3}{22}$ \\
$\bar{m}_{b} \leq \mu<M_{W}$ & $\frac{21}{44}$ & $\frac{15}{22}$ & $\frac{51}{440}$ & $\frac{3}{22}$ \\
$m_{\tau} \leq \mu<\bar{m}_{b}$ & $\frac{9}{20}$ & $\frac{3}{5}$ & $\frac{2}{19}$ & $\frac{1}{5}$ \\
$\bar{m}_{c} \leq \mu<m_{\tau}$ & $\frac{9}{20}$ & $\frac{2}{5}$ & $\frac{7}{80}$ & $\frac{1}{5}$ \\
$\bar{m}_{s} \leq \mu<\bar{m}_{c}$ & $\frac{1}{2}$ & $\frac{1}{2}$ & $\frac{5}{36}$ & 0 \\
$\bar{m}_{d} \leq \mu<\bar{m}_{s}$ & $\frac{9}{20}$ & $\frac{2}{5}$ & $\frac{13}{110}$ & $\frac{1}{20}$ \\
$\bar{m}_{u} \leq \mu<\bar{m}_{d}$ & $\frac{3}{8}$ & $\frac{1}{4}$ & $\frac{3}{40}$ & 0 \\
$m_{\mu} \leq \mu<\bar{m}_{u}$ & $\frac{1}{4}$ & 0 & 0 & 0 \\
$m_{e} \leq \mu<m_{\mu}$ & $\frac{1}{4}$ & 0 & 0 & 0 \\
\hline
\end{tabular}

Table 2. Coefficients entering the higher order RGE for the weak mixing angle.

with $n_{q}$ the number of active quarks and $N_{i}^{c}=3$ the color factor for quarks. For leptons one substitutes $N_{i}^{c}=1$ and $\hat{\alpha}_{s}=0$, while $K_{i}=1$ for bosons.

We can relate the RGE of $\hat{\alpha}$ to that of $\sin ^{2} \hat{\theta}_{W}$ since both, the $\gamma Z$ mixing tensor $\hat{\Pi}_{\gamma Z}$ and the photon vacuum polarization function $\hat{\Pi}_{\gamma \gamma}$ are pure vector-current correlators. Including higher order corrections, the RGE for the $Z$ boson vector coupling to fermion $f$, $\hat{v}_{f}=T_{f}-2 Q_{f} \sin ^{2} \hat{\theta}_{W}$, where $T_{f}$ is the third component of weak isospin of fermion $f$, is then

$$
\mu^{2} \frac{d \hat{v}_{f}}{d \mu^{2}}=\frac{\hat{\alpha} Q_{f}}{24 \pi}\left[\sum_{i} K_{i} \gamma_{i} \hat{v}_{i} Q_{i}+12 \sigma\left(\sum_{q} Q_{q}\right)\left(\sum_{q} \hat{v}_{q}\right)\right] .
$$

Eqs. (2.1) and (2.4) can be used [2] to obtain

$$
\begin{aligned}
\hat{s}^{2}(\mu)= & \hat{s}^{2}\left(\mu_{0}\right) \frac{\hat{\alpha}(\mu)}{\hat{\alpha}\left(\mu_{0}\right)}+\lambda_{1}\left[1-\frac{\hat{\alpha}(\mu)}{\hat{\alpha}\left(\mu_{0}\right)}\right]+ \\
& +\frac{\hat{\alpha}(\mu)}{\pi}\left[\frac{\lambda_{2}}{3} \ln \frac{\mu^{2}}{\mu_{0}^{2}}+\frac{3 \lambda_{3}}{4} \ln \frac{\hat{\alpha}(\mu)}{\hat{\alpha}\left(\mu_{0}\right)}+\tilde{\sigma}\left(\mu_{0}\right)-\tilde{\sigma}(\mu)\right],
\end{aligned}
$$

where the $\lambda_{i}$ are known [2] constants given in table 2 and the explicit $K_{i}$ dependence has disappeared. The $\tilde{\sigma}$ terms,

$$
\tilde{\sigma}(\mu)=\frac{\lambda_{4}}{33-2 n_{q}} \frac{5}{36}\left[\left(11-24 \zeta_{3}\right) \frac{\hat{\alpha}_{s}^{2}(\mu)}{\pi^{2}}+b \frac{\hat{\alpha}_{s}^{3}(\mu)}{\pi^{3}}\right]
$$


with,

$$
\begin{aligned}
b \equiv & \frac{2213}{24}-\frac{6955}{24} \zeta_{3}+\frac{99}{2} \zeta_{4}+\frac{775}{6} \zeta_{5}-n_{q}\left(\frac{55}{12}-\frac{41}{4} \zeta_{3}+3 \zeta_{4}+5 \zeta_{5}\right) \\
& -\frac{\left(153-19 n_{q}\right)\left(11-24 \zeta_{3}\right)}{99-6 n_{q}},
\end{aligned}
$$

represent the singlet contributions to the RGE evolution of the weak mixing angle at four and five loop order. These terms arise from quark-antiquark annihilation (disconnected) diagrams (see figure 1) and are suppressed in perturbative QCD (PQCD). In the nonperturbative domain these give rise to so-called OZI-rule [20-22] violations.

Eq. (2.5) together with the solution of the four-loop QCD $\beta$-function $[26,27]$ represents a complete solution, as long as all matching scales $\mu$ at which an active particle decouples are known, because there the $\lambda_{i}$ change their values. The matching scales of all bosons [28], charged leptons, and heavy $(t, b$, and $c$ ) quarks [29-31] can be calculated as what we call threshold masses $\bar{m}_{q}$, where the QCD corrections to the matching relations vanish by definition.

\subsection{Matching conditions}

At each particle threshold the RGE coefficients need to be modified to reflect the particle content of the associated effective field theory (EFT), and in the $\overline{\mathrm{MS}}$ scheme it is also convenient to change the definitions of $\hat{\alpha}$ and $\hat{s}$ to correspond to this same EFT. This is analogous to the usual treatment of $\hat{\alpha}_{s}$ and leads to very small matching discontinuities in the RGE running of the couplings.

Denoting the electromagnetic coupling with and without the fermion near the threshold by $\hat{\alpha}\left(m_{f}\right)^{+}$and $\hat{\alpha}\left(m_{f}\right)^{-}$, respectively, ${ }^{2}$ the matching condition for $\hat{\alpha}$ reads [29-31],

$$
\begin{aligned}
& \frac{\pi}{\hat{\alpha}\left(m_{f}\right)^{+}}= \frac{\pi}{\hat{\alpha}\left(m_{f}\right)^{-}}-\frac{15}{16} N_{f}^{c} \frac{\hat{\alpha}\left(m_{f}\right)}{\pi} Q_{f}^{4} \\
&-\frac{N_{f}^{c}-1}{2}\left[\frac{13}{12} \frac{\hat{\alpha}_{s}^{+}}{\pi}+\left(\frac{655}{144} \zeta_{3}-\frac{3847}{864}+\frac{361}{1296} n_{q}\right) \frac{\hat{\alpha}_{s}^{+2}}{\pi^{2}}\right. \\
&\left.+\left(-0.55739-0.92807 n_{q}+0.01928 n_{q}^{2}\right) \frac{\hat{\alpha}_{s}^{+3}}{\pi^{3}}\right] Q_{f}^{2} \\
&-\frac{N_{f}^{c}-1}{2}\left[\frac{295}{1296} \frac{\hat{\alpha}_{s}^{+2}}{\pi^{2}}+\left(\mathcal{K}_{1}+\mathcal{K}_{2} n_{q}\right) \frac{\hat{\alpha}_{s}^{+3}}{\pi^{3}}\right] \sum_{\ell} Q_{\ell}^{2} .
\end{aligned}
$$

The first three lines derive from heavy quark vector-current correlators. The last line involves a sum over all quarks $\ell$ with $m_{\ell} \ll m_{q}$, and arises from the decoupling of the heavy quark $q$ propagating in inner loops of multi-bubble type diagrams in which the outer loop (the one coupled to the currents) is occupied by a light quark $\ell$. The corresponding contribution at order $\hat{\alpha}_{s}^{3}$ is parametrized by the coefficients $\mathcal{K}_{i}$ and is unknown at present. The known $\hat{\alpha}_{s}^{2}$ term for the charm and bottom quarks, and the $\hat{\alpha}_{s}^{3}$ terms from the charm

\footnotetext{
${ }^{2}$ We assume $m_{f}$ is an $\overline{\mathrm{MS}}$ mass with respect to QCD, but a pole mass for both leptons and quarks with respect to QED.
} 

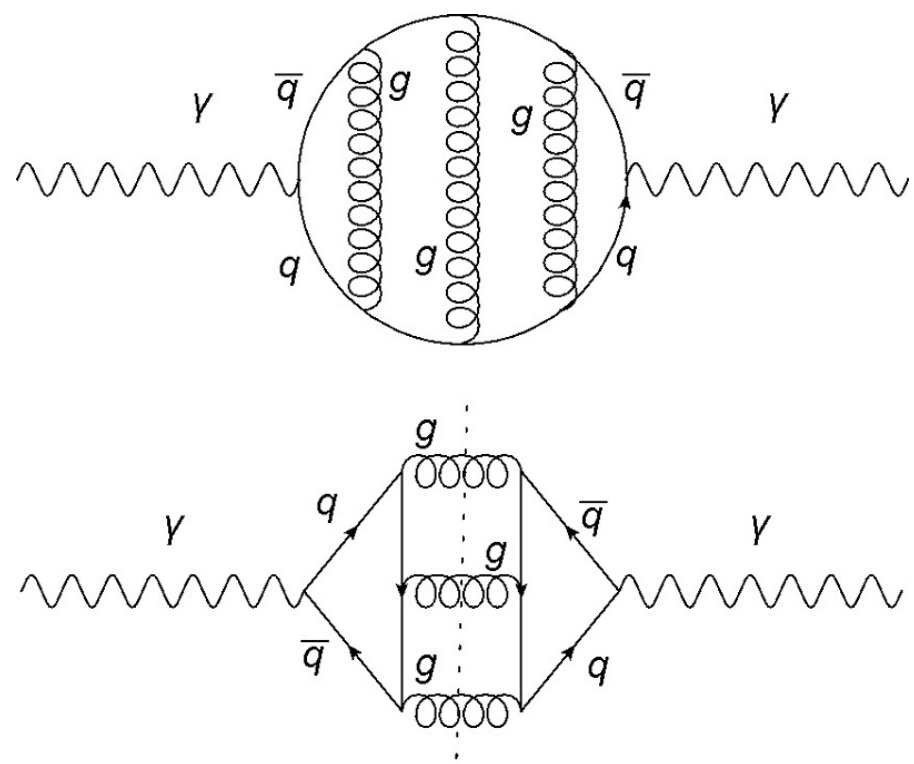

Figure 1. Examples of a connected (top) and a disconnected (bottom) Feynman diagram.

and bottom quark vector-current correlators amount to about $9 \times 10^{-6}$ and $-9 \times 10^{-6}$, respectively. Taking these as conservative bounds on the unknown higher-order terms and combining them in quadrature results in an estimated truncation error of $\pm 1.3 \times 10^{-5}$ in $\hat{\alpha}$.

The matching conditions of $\hat{s}^{2}$ and $\hat{\alpha}$ can also be related [2],

$$
\sin ^{2} \hat{\theta}_{W}\left(\hat{m}_{f}\right)^{-}=\frac{\hat{\alpha}\left(\hat{m}_{f}\right)^{-}}{\hat{\alpha}\left(\hat{m}_{f}\right)^{+}} \sin ^{2} \hat{\theta}_{W}\left(\hat{m}_{f}\right)^{+}+\frac{Q_{i} T_{i}}{2 Q_{i}^{2}}\left[1-\frac{\hat{\alpha}\left(\hat{m}_{f}\right)^{-}}{\hat{\alpha}\left(\hat{m}_{f}\right)^{+}}\right] .
$$

Applying the numerical analysis of the previous paragraph to eq. (2.9), we find $2.4 \times 10^{-6}$ and $-1.4 \times 10^{-6}$, respectively, and we estimate a truncation error related to the matching of about $\pm 3 \times 10^{-6}$ in $\hat{s}^{2}$.

For completeness we recall that integrating out the $W^{ \pm}$bosons induces the one-loop matching condition $[2,28]$,

$$
\frac{1}{\hat{\alpha}^{+}}=\frac{1}{\hat{\alpha}^{-}}+\frac{1}{6 \pi} .
$$

For $\hat{s}^{2}$ this implies

$$
\sin ^{2} \hat{\theta}_{W}\left(M_{W}\right)^{+}=1-\frac{\hat{\alpha}\left(M_{W}\right)^{+}}{\hat{\alpha}\left(M_{W}\right)^{-}} \cos ^{2} \hat{\theta}_{W}\left(M_{W}\right)^{-} .
$$

\section{Implementation of experimental input}

The perturbative treatment of the previous section cannot be applied at hadronic energy scales and experimental input is required. This is usually taken from $R(s)$, i.e., the cross section $\sigma\left(e^{+} e^{-} \rightarrow\right.$ hadrons) normalized to $\sigma\left(e^{+} e^{-} \rightarrow \mu^{+} \mu^{-}\right)$. Additional information on $R(s)$ is encoded in hadronic $\tau$ decay spectral functions [32]. The traditional method to 
implement the $R(s)$ measurements is through a subtracted dispersion integral,

$$
\Delta \alpha_{\text {had }}^{(5)}\left(M_{Z}^{2}\right)=\frac{\alpha}{3 \pi} \int_{4 m_{\pi}^{2}}^{\infty} d s \frac{R(s) M_{Z}^{2}}{s\left(M_{Z}^{2}-s\right)-i \epsilon},
$$

which gives the hadronic contribution (with the top quark removed) to the $Z$ scale value of the electromagnetic coupling in the on-shell scheme. One supplements the input data with the theoretical (perturbative) prediction for $R(s)$ at $s \geq s_{0}$, with $s_{0}$ large enough to be able to trust QCD perturbation theory. A variant [33] of this approach evaluates eq. (3.1) in the space-like region, $\Delta \alpha_{\text {had. }}^{(5)}\left(-M_{Z}^{2}\right)$, and obtains $\Delta \alpha_{\text {had. }}^{(5)}\left(M_{Z}^{2}\right)$ in a second step. More details about how different groups get the running of alpha are given in appendix A.

In the $\overline{\mathrm{MS}}$ scheme it is more natural to use an unsubstracted dispersion relation [24],

$$
\Delta \hat{\alpha}^{(3)}\left(\mu_{0}\right)=\frac{\alpha}{3 \pi} \int_{4 m_{\pi}^{2}}^{\mu_{0}^{2}} d s \frac{R(s)}{s-i \epsilon}+4 \pi I^{(3)},
$$

where the superscript indicates that we focus here on the currents produced by the three light quarks (bosons, leptons, charm and bottom quarks are included following section 2). The upper integration limit can in principle be chosen as an arbitrary perturbative scale $\mu_{0}$, but in practice we take $\mu_{0}^{2}$ to coincide with the cut-off value $s_{0}$ used in the traditional method, since this allows us to recycle results obtained there. Indeed [24],

$$
\frac{\alpha}{3 \pi} \int_{4 m_{\pi}^{2}}^{\mu_{0}^{2}} d s\left[\frac{R(s)}{s-i \epsilon}-\frac{R(s) M_{Z}^{2}}{s\left(M_{Z}^{2}-s\right)-i \epsilon}\right]<10^{-6},
$$

for $\mu_{0} \lesssim 2 \mathrm{GeV}$. Using the results of ref. [16] including inputs from $\tau$ decays which we correct for $\gamma-\rho$ mixing [17], we obtain,

$$
\frac{\alpha}{3 \pi} \int_{4 m_{\pi}^{2}}^{4 \mathrm{GeV}^{2}} d s \frac{R(s) M_{Z}^{2}}{s\left(M_{Z}^{2}-s\right)}=(58.71 \pm 0.45) \times 10^{-4} .
$$

We compute the second term in eq. (3.2) at the scale $\mu=2 \mathrm{GeV}$ perturbatively [34], extending the $\mathcal{O}\left(\hat{\alpha}_{s}^{2}\right)$ result of ref. [24] to $\mathcal{O}\left(\hat{\alpha}_{s}^{3}\right)$,

$$
\begin{aligned}
4 \pi I^{(3)}=2 \alpha \int_{0}^{2 \pi} d \theta \hat{\Pi}^{(3)}\left(\mu^{2} e^{i \theta}\right) & \\
=\frac{2 \alpha}{3 \pi}\left[\frac{5}{3}+\left(\frac{55}{12}-4 \zeta(3)+2 \frac{\hat{m}_{s}^{2}}{\mu^{2}}\right)\left(\frac{\hat{\alpha}_{s}}{\pi}+\frac{\hat{\alpha}}{4 \pi}\right)\right. & \\
+ & \left(\frac{34525}{864}-\frac{9}{4} \zeta(2)-\frac{715}{18} \zeta(3)+\frac{25}{3} \zeta(5)+\frac{125}{12} \frac{\hat{m}_{s}^{2}}{\mu^{2}}+F_{2}\left(\hat{m}_{c}, \hat{m}_{b}\right)\right) \frac{\hat{\alpha}_{s}^{2}}{\pi^{2}} \\
+ & \left(\frac{7012579}{13824}-\frac{961}{16} \zeta(2)-\frac{76681}{144} \zeta(3)+\frac{12515}{288} \zeta(5)\right. \\
& \left.\left.\quad-\frac{665}{36} \zeta(7)+\frac{81}{2} \zeta(2) \zeta(3)+\frac{155}{2} \zeta(3)^{2}+F_{3}\left(\hat{m}_{c}, \hat{m}_{b}\right)\right) \frac{\hat{\alpha}_{s}^{3}}{\pi^{3}}\right] \\
=(24.85 \pm & \left.0.18-43 \Delta \hat{\alpha}_{s}\right) \times 10^{-4},
\end{aligned}
$$


where the $F_{i}\left(\hat{m}_{c}, \hat{m}_{b}\right)$ are correction terms from the charm and bottom quarks. The explicit analytical expression for $F_{2}\left(\hat{m}_{c}, \hat{m}_{b}\right) \simeq-0.2348$ is given in ref. [24], while that for $F_{3}\left(\hat{m}_{c}, \hat{m}_{b}\right) \simeq-0.390$ will appear in ref. [34]. The numerical evaluation in the last line of eq. (3.5) is for $\hat{\alpha}_{s}\left(M_{Z}\right)=0.1182, \hat{\alpha}_{s}(2 \mathrm{GeV})=0.303$ and $\hat{m}_{s}(2 \mathrm{GeV})=98 \pm 6 \mathrm{MeV}$ [35]. The uncertainty is the size of the $\mathcal{O}\left(\hat{\alpha}_{s}^{3}\right)$ term, and we have defined

$$
\Delta \hat{\alpha}_{s} \equiv \hat{\alpha}_{s}\left(M_{Z}\right)-0.1182
$$

to display the dependence on $\hat{\alpha}_{s}$. Thus, from eqs. (3.2)-(3.5) we obtain,

$$
\Delta \hat{\alpha}^{(3)}(2 \mathrm{GeV})=(83.56 \pm 0.45 \pm 0.18) \times 10^{-4} .
$$

\section{Singlet contribution}

We recall that eq. (2.6) exhibits an explicit dependence on $\alpha_{s}$, which in the non-perturbative domain gives rise to the QCD induced OZI-rule [20-22] violations. These have to be known independently, since they affect $\hat{\alpha}$ and $\hat{s}^{2}$ differently. Thus, in addition to a quark flavor separation, one also needs a singlet piece separation, even though the singlet piece is expected to be small. To do so, we first relate $\Delta_{\text {disc }} \hat{\alpha}$, the disconnected part in $\Delta \hat{\alpha}^{(3)}(2 \mathrm{GeV})$, to the one entering the low energy weak mixing angle, $\Delta_{\text {disc }} \hat{s}^{2}$. Non-singlet and singlet contributions are separately gauge-invariant, and to gain information on $\Delta_{\text {disc }} \hat{\alpha}$, we will adopt a lattice QCD calculation [23] of the disconnected quark line contributions to the anomalous magnetic moment of the muon, $a_{\mu}$.

By construction, the $\tilde{\sigma}$ terms in eq. (2.5) are related to the $\sigma$ terms in eq. (2.1),

$$
\mu^{2} \frac{d \tilde{\sigma}}{d \mu^{2}}=-\lambda_{4} \sigma
$$

On the other hand, isolating the $\Delta_{\text {disc }} \hat{\alpha}$ term in eq. (2.1) we obtain (working here in lowest order in $\alpha$ ),

$$
\mu^{2} \frac{d \Delta_{\text {disc }} \hat{\alpha}}{d \mu^{2}}=\frac{\alpha}{\pi}\left(\sum_{q} Q_{q}\right)^{2} \sigma
$$

so that,

$$
\frac{d \tilde{\sigma}}{d \mu^{2}}=-\frac{\pi}{\alpha} \lambda_{4}\left(\sum_{q} Q_{q}\right)^{-2} \frac{d \Delta_{\text {disc }} \hat{\alpha}}{d \mu^{2}}=-\lambda_{1} \frac{\pi}{\alpha} \frac{d \Delta_{\text {disc }} \hat{\alpha}}{d \mu^{2}},
$$

where the last step applies for $\mu<\bar{m}_{c}$ (we are assuming approximate isospin symmetry which eliminates the interval $\bar{m}_{u}<\mu<\bar{m}_{d}$ ). Then,

$$
\tilde{\sigma}(\mu)-\tilde{\sigma}\left(\mu_{0}\right)=-\lambda_{1} \frac{\pi}{\alpha}\left[\Delta_{\text {disc }} \hat{\alpha}(\mu)-\Delta_{\text {disc }} \hat{\alpha}\left(\mu_{0}\right)\right] .
$$

These relations are general, but there is a subtle point. In general, the singlet pieces effectively decouple at renormalization scales $\bar{m}_{q}^{\text {disc }}$ that may differ from the scales $\bar{m}_{q}$ at which the non-singlet pieces decouple. This would generate various energy intervals with generally different values for $\lambda_{1}$. Implementing strong isospin symmetry in the form 
$\bar{m}_{u}=\bar{m}_{d}$ and $\bar{m}_{u}^{\text {disc }}=\bar{m}_{d}^{\text {disc }}$, as well as accepting the physical mass orderings $\bar{m}_{s} \geq \bar{m}_{u}$ and $\bar{m}_{s}^{\text {disc }} \geq \bar{m}_{u}^{\text {disc }}$, there remain a total of six different orderings.

As an example, consider the case,

$$
\bar{m}_{s}^{\text {disc }}>\bar{m}_{s}>\bar{m}_{u}>\bar{m}_{u}^{\text {disc }}
$$

For scales $\mu>\bar{m}_{s}^{\text {disc }}$ there are three active quarks with $Q_{u}+Q_{d}+Q_{s}=0$ and the singlet contributions vanish. For scales in the range $\bar{m}_{s}^{\text {disc }}>\mu>\bar{m}_{s}$ we obtain the value $\lambda_{1}=1 / 2$. Similarly, for $\bar{m}_{s}>\mu>\bar{m}_{u}$ and for $\bar{m}_{u}>\bar{m}_{u}^{\text {disc }}$ we find $\lambda_{1}=9 / 20$ and $1 / 4$, respectively. Below $\bar{m}_{u}^{\text {disc }}$ all singlet contributions vanish by definition. Inserting these results into eq. (4.4) and summing the contributions from all intervals, we find the constraint,

$$
-\frac{\Delta_{\text {disc }} \hat{\alpha}}{4}<\frac{\alpha}{\pi}\left[\tilde{\sigma}\left(\bar{m}_{s}^{\text {disc }}\right)-\tilde{\sigma}\left(\bar{m}_{u}^{\text {disc }}\right)\right]<-\frac{\Delta_{\text {disc }} \hat{\alpha}}{2},
$$

where we have anticipated that $\Delta_{\text {disc }} \hat{\alpha}<0$ (see below).

The other five cases are dealt with in the same way, and one can check that the inequality (4.6) is never violated. For the three mass orderings satisfying $\bar{m}_{u}^{\text {disc }} \geq \bar{m}_{u}$, or generally if we can neglect the presumably small range $\bar{m}_{u}>\mu>\hat{m}_{u}^{\text {disc }}$, we find the much stronger constraint,

$$
-\frac{9 \Delta_{\text {disc }} \hat{\alpha}}{20}<\frac{\alpha}{\pi}\left[\tilde{\sigma}\left(\bar{m}_{s}^{\text {disc }}\right)-\tilde{\sigma}\left(\bar{m}_{u}^{\text {disc }}\right)\right]<-\frac{\Delta_{\text {disc }} \hat{\alpha}}{2} .
$$

Since we do not expect the $\bar{m}_{q}^{\text {disc }}$ to be numerically very different from the $\bar{m}_{q}$ we choose our central value to correspond to $\bar{m}_{q}^{\text {disc }}=\bar{m}_{q}$, and we include twice the range in eq. (4.7) as the uncertainty due to possible $\bar{m}_{q}^{\text {disc }} \neq \bar{m}_{q}$ effects. Thus,

$$
\frac{\alpha}{\pi}\left[\tilde{\sigma}\left(\bar{m}_{s}^{\text {disc }}\right)-\tilde{\sigma}\left(\bar{m}_{u}^{\text {disc }}\right)\right]=-\left[\frac{9}{20} \pm \frac{1}{20}\right] \Delta_{\text {disc }} \hat{\alpha}
$$

which can be inserted into eq. (2.5). Notice, however, that eq. (2.5) also contains an implicit singlet contribution from each of the two terms in the first line. Taken together, the $\lambda_{1}$ term cancels exactly the central value in eq. (4.8) and we finally arrive at

$$
\Delta_{\text {disc }} \hat{s}^{2}=\left[\hat{s}^{2} \pm \frac{1}{20}\right] \Delta_{\text {disc }} \hat{\alpha} .
$$

In appendix B we compute $\Delta_{\text {disc }} \alpha$ in the on-shell scheme by exploiting the lattice gauge theory calculation [23] of the corresponding contribution to $a_{\mu}$ with the result,

$$
\Delta_{\text {disc }} \alpha(2.0 \mathrm{GeV})=-2.6 \times 10^{-5} .
$$

Note that because the sum of the charges of the three light quarks vanishes, and we enter the perturbative domain where the singlet piece is known to be tiny, we expect an asymptotically stable value at higher energies for $\Delta_{\text {disc }} \alpha(q)$. This is supported by figure 2 , showing that $\Delta_{\text {disc }} \alpha(q)$ is nearly $q$-independent for $q \gtrsim 1.2 \mathrm{GeV}$. We also remark that the dominance of low scales notwithstanding, the sign in eq. (4.10) coincides with that of the singlet piece 


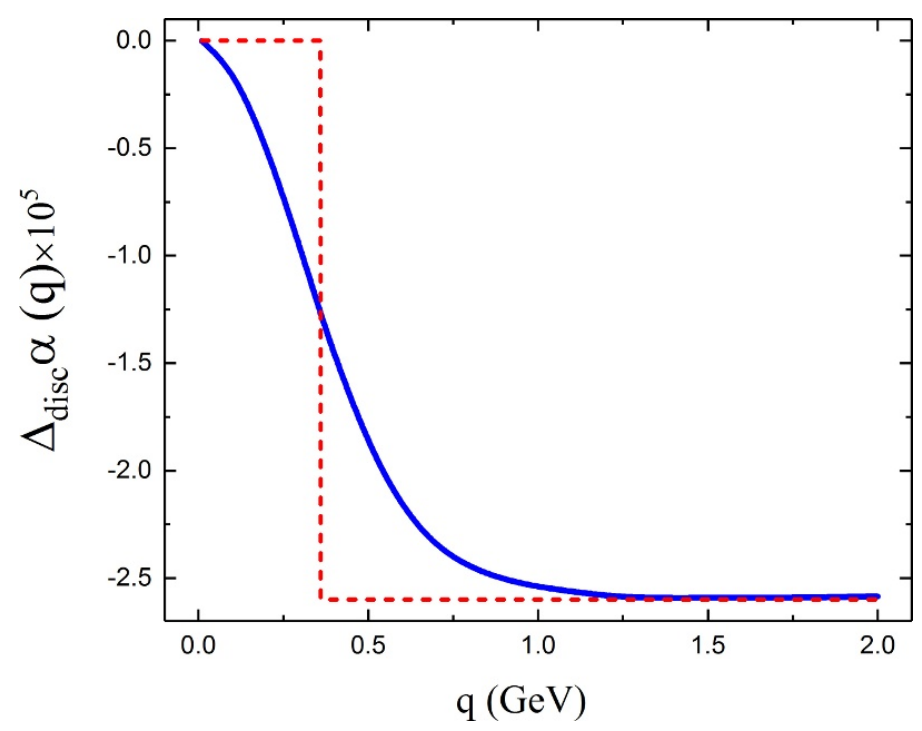

Figure 2. Scale dependence of the singlet contribution to $\Delta \alpha$ (solid line) and its step function approximation (dashed line).

in the perturbative regime. Also shown in figure 2 is the step function approximation of $\Delta_{\text {disc }} \alpha(q)$, with the step defined as the value of $q$ where it reaches half of its asymptotic value in eq. (4.10). We interpret this as the value where the strange quark decouples from singlet diagrams, so that $\bar{m}_{s}^{\text {disc }} \sim 350 \mathrm{MeV}$. Our central value of $\bar{m}_{s}$ to be derived in the next section, $\bar{m}_{s}=342 \mathrm{MeV}$, is numerically very close to this providing evidence for $\bar{m}_{s}^{\text {disc }} \approx \bar{m}_{s}$.

Eq. (4.9) and eq. (4.10) refer to quantities in the $\overline{\mathrm{MS}}$ and on-shell schemes, respectively, and in general these may differ. However, since we are working here in the three quark theory and the sum of the charges of three light quarks vanishes, the change of schemes is trivial. We can therefore use eq. (4.10) in eq. (4.9) and obtain,

$$
\Delta_{\text {disc }} \hat{s}^{2}=(-0.6 \pm 0.3) \times 10^{-5},
$$

where the uncertainty combines the errors from eq. (4.9) and the one induced by the lattice calculation [23].

\section{Flavor separation}

In this section we perform a flavor separation of the contributions of up-type from downtype quarks, or - given that up and down quarks are linked by the approximate strong isospin symmetry - a separation of $s$ from $u$ and $d$ quarks. Our strategy consists of first using exclusively the experimental electro-production data as tabulated in ref. [16] to constrain the contribution $\Delta_{s} \alpha$ of the strange quark to $\Delta \alpha$. We then exploit the lattice gauge theory results in refs. $[18,19]$ to confirm and refine the purely data driven analysis. Then we introduce the threshold mass $\bar{m}_{q}$ of a quark $q$ as the value of the 't Hooft scale where the QCD contribution to the corresponding decoupling relation becomes trivial. $\bar{m}_{c}$ and $\bar{m}_{b}$ are treated in perturbation theory, while for $u, d$, and $s$ quarks we derive bounds using phenomenological and theoretical constraints. 


\begin{tabular}{|lrr|}
\hline channel & $a_{\mu} \times 10^{10}$ & $\Delta \alpha \times 10^{4}$ \\
\hline$\phi$ & 38.43 & 5.13 \\
$K \bar{K} \pi$ & 2.45 & 0.78 \\
$\eta \phi$ & 0.36 & 0.13 \\
PQCD $[36](>1.8 \mathrm{GeV})$ & 7.30 & - \\
\hline Total & 48.54 & 6.04 \\
\hline$K \bar{K}($ non $-\phi)$ & 3.62 & 0.76 \\
$K \bar{K} 2 \pi$ & 0.85 & 0.30 \\
$K \bar{K} 3 \pi$ & -0.03 & -0.01 \\
$K \bar{K} \eta$ & 0.01 & 0.00 \\
$K \bar{K} \omega$ & 0.01 & 0.00 \\
\hline Total & 4.46 & 1.05 \\
\hline
\end{tabular}

Table 3. Channels associated with the strange quark external current (top) and possible further channels originating from it (bottom).

\subsection{Experimental data}

To obtain $\Delta_{s} \alpha$ we use ref. [16] where the contribution of each hadronic channel to $a_{\mu}$ and $\Delta \alpha$ for energies up to $1.8 \mathrm{GeV}$ is given. The main idea is to determine for each channel whether it was produced by an $\bar{s} s$ or a first generation quark current. For reasons that will become clear later, we consider both, $\Delta_{s} \alpha$, and the strange quark contribution to the anomalous magnetic moment, $a_{\mu}^{s}$.

We begin by listing in the upper part of table 3 the experimental channels [16] which we associate with an $s \bar{s}$ current. Up to OZI-rule violating $\phi-\omega$ and $\phi-\rho$ mixing effects, the $\phi$ meson can be identified with strange quarks. We calculate its contribution using a BreitWigner shape with $s$-dependent total and partial widths, adopting the PDG values [35] for the $\phi$ meson branching ratios and applying a small correction for $\phi-\omega$ mixing. As for the $\phi(1680)$, the main decay channel is $K \bar{K}^{*}$ with $K^{*}$ mesons decaying almost entirely into $K \pi$. As can be seen from data [16], the $K \bar{K} \pi$ channel is indeed virtually saturated by $\phi(1680)$ decays. The $\eta-\phi$ channel also arises dominantly from the strange quark current since the contribution to this channel from light quarks is Zweig rule suppressed. Conversely, we expect channels involving an $\eta$ meson accompanied by non-strange states to be mainly due to light quark currents. For $a_{\mu}^{s}$ we need to add the contribution from energies above 1.8 GeV. It can be computed within PQCD and taken as one sixth of the corresponding light quark contribution [36] of $43.8 \times 10^{-10}$. The lower part of table 3 shows further channels involving strange quarks to which first generation quark currents could conceivably contribute, and we conservatively assign $(50 \pm 50) \%$ of these to the $s \bar{s}$ current. The table also shows the corresponding contributions to $a_{\mu}$. Adding the totals in this way we find,

$$
a_{\mu}^{s}=(50.77 \pm 0.60 \pm 0.83 \pm 2.23) \times 10^{-10}=(50.77 \pm 2.45) \times 10^{-10},
$$


and,

$$
\Delta_{s} \alpha(1.8 \mathrm{GeV})=(6.56 \pm 0.11 \pm 0.19 \pm 0.53) \times 10^{-4}=(6.56 \pm 0.57) \times 10^{-4} .
$$

The first errors are experimental [16] where we accounted for correlations. The second errors allow for differences in parametrizations when decay parameters are extracted from experimental data by different groups. The last errors are half of the totals in table 3 , but we expect the $s \bar{s}$ current to virtually saturate the kaon channels in table 3 because the larger strange quark mass should suppress the probability amplitude to produce an $s \bar{s}$ sea quark pair relative to first generation quark pairs.

The uncertainty in eq. (5.2) is already about three times smaller than in the past [2]. We can reduce it further by quantifying our expectation that the strange quark current actually saturates the kaon channels listed in the bottom part of table 3. For this, we re-write eqs. (5.1) and (5.2) in the form,

$$
\begin{aligned}
a_{\mu}^{s} & =(53.00-4.46 \kappa \pm 0.60 \pm 0.83) \times 10^{-10}, \\
\Delta_{s} \alpha(1.8 \mathrm{GeV}) & =(7.09-1.05 \kappa \pm 0.11 \pm 0.19) \times 10^{-4},
\end{aligned}
$$

with a parameter $0 \leq \kappa \leq 1$, where $\kappa=0(\kappa=1)$ corresponds to the case where all kaon contributions in table 3 arise from the strange (first generation) quark current. In order to confirm that indeed $\kappa \approx 0$ and to compute an uncertainty for possible $\kappa \neq 0$ effects, we can use results on $a_{\mu}^{s}$ from lattice gauge theory, as we show next.

\subsection{Lattice data}

Two groups $[18,19]$ calculated the contribution of the strange quark to the vacuum polarization function within lattice gauge theory with a focus on $a_{\mu}^{s}$. The two results agree and average to

$$
a_{\mu}^{s}=(53.32 \pm 0.49) \times 10^{-10} \quad \text { [lattice] }
$$

which is in perfect agreement with eq. (5.3) and our expectation $\kappa \approx 0$. Since the analogous result for $\Delta_{s} \alpha(1.8 \mathrm{GeV})$ has not been provided by either of the groups, we follow a Bayesian procedure to quantify the parameter $\kappa$ in eq. (5.4), using as prior information the comparison of eq. (5.3) with eq. (5.5). The $68.3 \%$ highest probability interval of $\kappa$, namely $0 \leq \kappa \leq \kappa_{1 \sigma}$, can be obtained from

$$
N \int_{0}^{\kappa_{1 \sigma}} \exp \left[-\frac{(53.00-4.46 \kappa-53.32)^{2}}{2\left(0.60^{2}+0.83^{2}+0.49^{2}\right)}\right] d \kappa=0.683
$$

where $N$ is the normalization of the distribution. This yields $\kappa_{1 \sigma}=0.22$, and eq. (5.4) now provides us with the desired result,

$$
\Delta_{s} \alpha(1.8 \mathrm{GeV})=(7.09 \pm 0.11 \pm 0.19 \pm 0.23) \times 10^{-4}=(7.09 \pm 0.32) \times 10^{-4},
$$

which is consistent with, but more precise than eq. (5.2). We assigned the uncertainty from $\kappa_{1 \sigma}$ symmetrically around $\kappa=0$, which is both the physically favored and most probable 
value (the peak of the distribution). This rather conservative treatment effectively doubles the error from $\kappa_{1 \sigma}$, and is meant to account for the fact that the kernels of $\Delta \alpha$ and $a_{\mu}$ differ.

The experimental values of $a_{\mu}$ and $\Delta \alpha$ are correlated, possibly impacting eq. (5.7). However, we found that even assuming them to be fully correlated changes the central value only very slightly and reduces the uncertainty modestly. Thus, we keep eq. (5.7) as our final result on $\Delta_{s} \alpha(1.8 \mathrm{GeV})$. As an additional cross-check we used the vacuum polarization function of another lattice calculation [19] of $a_{\mu}^{s}$ (expressed as a Padé approximant which is the source of the largest uncertainty [19]) to first reproduce their results, and then we computed $\Delta_{s} \alpha$ which yields,

$$
\Delta_{s} \alpha(1.8 \mathrm{GeV}) \approx(6.9 \pm 0.5) \times 10^{-4} \quad \text { [lattice] }
$$

in excellent agreement with eq. (5.7).

\subsection{Threshold masses}

\subsubsection{Heavy quarks}

We can compute $\bar{m}_{c}$ and $\bar{m}_{b}$ in perturbation theory by reincorporating the RGE summable logarithms of the form $\ln \hat{m}_{q} / \bar{m}_{q}$ into eq. (2.8), and then solving for $\bar{m}_{q}$ by setting the contribution from quark $q$ equal to zero. Since $\bar{m}_{q} \rightarrow \hat{m}_{q}$ for $\hat{\alpha}_{s} \rightarrow 0$, these logarithms are at most of order $\hat{\alpha}_{s}$ and can be ignored in the $\hat{\alpha}_{s}^{3}$ coefficient. Thus, we can use a previous analysis [24] where the logarithms up to order $\hat{\alpha}_{s}^{2}$ are given. We find,

$$
\begin{aligned}
\bar{m}=\hat{m}\{1 & -\frac{13}{24} \frac{\hat{\alpha}_{s}}{\pi}+\left(\frac{10073}{3456}-\frac{655}{288} \zeta_{3}-\frac{361}{2592} n_{q}\right) \frac{\hat{\alpha}_{s}^{2}}{\pi^{2}} \\
+ & \left(1.61024+0.59599 n_{q}-0.00964 n_{q}^{2}\right) \frac{\hat{\alpha}_{s}^{3}}{\pi^{3}} \\
+ & {\left.\left[-\frac{295}{2592} \frac{\hat{\alpha}_{s}^{2}}{\pi^{2}}+\left(\frac{5767}{62208}-\frac{\mathcal{K}_{1}+\mathcal{K}_{2} n_{q}}{2}\right) \frac{\hat{\alpha}_{s}^{3}}{\pi^{3}}\right] \frac{\sum Q_{\ell}^{2}}{Q_{h}^{2}}\right\} . }
\end{aligned}
$$

Using the input values for the $Z$ boson mass [35], $M_{Z}=91.1876 \mathrm{GeV}$, the charm quark mass [37], $\hat{m}_{c}\left(\hat{m}_{c}\right)=1.272 \mathrm{GeV}$, and the bottom quark mass [35], $\hat{m}_{b}\left(\hat{m}_{b}\right)=4.18 \mathrm{GeV}$, together with the 4-loop RGE [26] for $\hat{\alpha}_{s}$ with $n_{q}=4$ and $n_{q}=5$, respectively, we find

$$
\begin{aligned}
\bar{m}_{c} & =1.185 \mathrm{GeV}, \\
\bar{m}_{b} & =3.990 \mathrm{GeV} .
\end{aligned}
$$

It will be useful for later to define quantities $\xi_{q}[2]$ as ratios between the threshold mass of quark $q$ and the $1 S \bar{q} q$ bound state mass,

$$
\xi_{q} \equiv \frac{2 \bar{m}_{q}}{M_{1 S}} .
$$

This definition implies that $\xi_{q} \rightarrow 1$ for $\bar{m}_{q} \rightarrow \infty$ and $\xi_{q} \rightarrow 0$ for $\bar{m}_{q} \rightarrow 0$. We expect $\xi_{q}$ to be a monotonically increasing in the sense that $\xi_{1}>\xi_{2}$ if $\bar{m}_{1}>\bar{m}_{2}$. Using the PDG values for the bound state masses [35] we find $\xi_{c}=0.766$ and $\xi_{b}=0.844$, and thus $\xi_{b}>\xi_{c}$ as expected. 


\subsubsection{Light quarks}

Next we constrain the individual contributions of the light quarks to $\Delta \hat{\alpha}$, evaluated at $\bar{m}_{c}$. Using the RGE and the starting value given in eq. (3.7) we obtain,

$$
\Delta \hat{\alpha}^{(3)}\left(\bar{m}_{c}\right)=(65.10 \pm 0.45 \pm 0.18) \times 10^{-4} .
$$

From eq. (5.7) we can also calculate $\Delta_{s} \hat{\alpha}$ at $\bar{m}_{c}$. To do so, we first invoke experimental data to obtain the shift,

$$
\Delta_{s} \alpha(2 \mathrm{GeV})=\Delta_{s} \alpha(1.8 \mathrm{GeV})+(0.55 \pm 0.04) \times 10^{-4},
$$

given by one sixth of the continuum contribution [16] of $(3.31 \pm 0.26) \times 10^{-4}$ between the two scales. The uncertainty is the difference to using PQCD instead of data and accounts for quark-hadron duality violations. Changing to the $\overline{\mathrm{MS}}$ scheme and employing again the RGE gives,

$$
\Delta_{s} \hat{\alpha}\left(\bar{m}_{c}\right)=(8.71 \pm 0.32) \times 10^{-4} .
$$

Since the threshold mass is the value of the 't Hooft scale corresponding to trivial matching conditions regarding the QCD contribution, we can write,

$$
\Delta_{s} \hat{\alpha}\left(\bar{m}_{c}\right)=Q_{s}^{2} \frac{\alpha}{\pi} K_{\mathrm{QCD}}^{s}\left(\bar{m}_{c}\right) \ln \frac{\bar{m}_{c}^{2}}{\bar{m}_{s}^{2}},
$$

where we defined a scale dependent factor $K_{\mathrm{QCD}}^{q}(\mu)$ as the average QCD correction to the $\beta$ function between $\bar{m}_{q}$ and the scale $\mu$. Eq. (5.16) has two unknowns, $K_{\mathrm{QCD}}^{s}\left(\bar{m}_{c}\right)$ and $\bar{m}_{s}$, and it shows that increasing $K_{\mathrm{QCD}}^{s}\left(\bar{m}_{c}\right)$ forces the logarithm to decrease and in turn $\bar{m}_{s}$ to increase. Thus, smaller (larger) values of $K_{\mathrm{QCD}}^{s}\left(\bar{m}_{c}\right)$ correspond to a smaller (larger) values of $\bar{m}_{s}$. On the other hand, if we have two quarks with masses $\bar{m}_{1}>\bar{m}_{2}$, we expect the average QCD contribution between $\bar{m}_{2}$ and $\mu$ to be larger than that between $\bar{m}_{1}$ and $\mu$, since $\alpha_{s}$ is larger at lower scales. Thus,

$$
\bar{m}_{1}>\bar{m}_{2} \quad \Longrightarrow \quad K_{\mathrm{QCD}}^{1}(\mu)<K_{\mathrm{QCD}}^{2}(\mu),
$$

and we must have,

$$
K_{\mathrm{QCD}}^{c}\left(\bar{m}_{c}\right)<K_{\mathrm{QCD}}^{s}\left(\bar{m}_{c}\right) .
$$

$K_{\mathrm{QCD}}^{c}\left(\bar{m}_{c}\right)$ can be computed from eq. (2.2). Using $n_{q}=3$ and $\alpha_{s}\left(\bar{m}_{c}\right)=0.413$ yields $K_{\mathrm{QCD}}^{c}\left(\bar{m}_{c}\right)=1.178$, and implies the lower bound,

$$
\bar{m}_{s}>\bar{m}_{c} \exp \left[-\frac{\pi \Delta_{s} \hat{\alpha}\left(\bar{m}_{c}\right)}{2 Q_{s}^{2} \alpha K_{\mathrm{QCD}}^{c}\left(\bar{m}_{c}\right)}\right]=289 \mathrm{MeV},
$$

where we used $\alpha=\alpha\left(\bar{m}_{s}\right) \approx 1 / 135$. We can also obtain an upper bound on $\bar{m}_{s}$,

$$
\frac{2 \bar{m}_{s}}{M_{\phi}}=\xi_{s}<\xi_{c}=0.766 \quad \Longrightarrow \quad \bar{m}_{s}<390 \mathrm{MeV},
$$

implying $K_{\mathrm{QCD}}^{s}\left(\bar{m}_{c}\right)<1.50$. We can summarize these results by writing,

$$
K_{\mathrm{QCD}}^{s}\left(\bar{m}_{c}\right)=1.34 \pm 0.16, \quad \bar{m}_{s}=342_{-53}^{+48} \mathrm{MeV} .
$$




\begin{tabular}{|lc|}
\hline source & $\delta \sin ^{2} \hat{\theta}_{W}(0) \times 10^{5}$ \\
\hline$\Delta \hat{\alpha}^{(3)}(2 \mathrm{GeV})$ & 1.2 \\
flavor separation & 1.0 \\
isospin breaking & 0.7 \\
singlet contribution & 0.3 \\
PQCD & 0.6 \\
\hline Total & 1.8 \\
\hline
\end{tabular}

Table 4. Theoretical uncertainties in the low energy mixing angle.

$\bar{m}_{u}$ and $\bar{m}_{d}$ can be obtained in a similar way. We have,

$$
\Delta_{\mathrm{conn}} \hat{\alpha}^{(3)}\left(\bar{m}_{c}\right)=\Delta_{s} \hat{\alpha}\left(\bar{m}_{c}\right)+\frac{2 \alpha}{\pi}\left[\left(Q_{u}^{2}+Q_{d}^{2}\right) K_{\mathrm{QCD}}^{u, d} \ln \frac{\bar{m}_{c}}{\bar{m}_{u, d}}\right],
$$

where the quark connected contribution to $\Delta \hat{\alpha}^{(3)}\left(\bar{m}_{c}\right)$ is given by,

$$
\Delta_{\text {conn }} \hat{\alpha}^{(3)}\left(\bar{m}_{c}\right) \equiv \Delta \hat{\alpha}^{(3)}\left(\bar{m}_{c}\right)-\Delta_{\text {disc }} \hat{\alpha}^{(3)}\left(\bar{m}_{c}\right)=65.36 \times 10^{-4} .
$$

Following the same steps as for $\bar{m}_{s}$ we find,

$$
K_{\mathrm{QCD}}^{u, d}\left(\bar{m}_{c}\right)=1.38 \pm 0.20, \quad \bar{m}_{u, d}=246_{-57}^{+54} \mathrm{MeV},
$$

where the errors in eqs. (5.21) and (5.24) are strongly correlated. The light quark threshold masses are convenient for implementing the RGE and serve an illustrative purpose, but their precise values affect $\hat{s}(0)$ only at order $\mathcal{O}\left(\alpha^{2}\right)$ and beyond, as long as the central value in eq. (5.15) remains fixed (the uncertainty there will give rise to the flavor separation error). Notice, that for the central values we have $\bar{m}_{s}-\bar{m}_{u} \approx 96 \mathrm{MeV}$, which is of typical size for hadronic mass splittings within $\mathrm{SU}(3)$ flavor multiplets.

Finally, accounting for the squares of the electric charges we obtain the contributions from the first generation quarks at the scale $\bar{m}_{s}$,

$$
\Delta \hat{\alpha}^{(2)}\left(\bar{m}_{s}\right)=\Delta \hat{\alpha}^{(3)}\left(\bar{m}_{c}\right)-6 \Delta_{s} \hat{\alpha}\left(\bar{m}_{c}\right)=(12.9 \mp 1.9) \times 10^{-4},
$$

where we only quote the uncertainty from the flavor separation in eq. (5.15).

\section{Theoretical uncertainties}

In addition to parametric uncertainties, there are five sources of theoretical uncertainties for the weak mixing angle at low energies affecting our calculation. They are summarized in table 4 and discussed in the following.

The first uncertainty is induced by the experimental error in the determination of $\Delta \hat{\alpha}^{(3)}(2.0 \mathrm{GeV})$. Eq. (2.5) propagates this uncertainty to the weak mixing angle [2],

$$
\delta \hat{s}^{2}(0)=\left[\frac{1}{2}-\hat{s}^{2}\right] \delta \Delta \hat{\alpha}^{(3)}(2 \mathrm{GeV})=\mp 1.2 \times 10^{-5},
$$

where we have used $\delta \Delta \hat{\alpha}^{(3)}(2 \mathrm{GeV})= \pm 0.45 \times 10^{-4}$ from eq. (3.4). 
The three light quarks enter with different electroweak weights into $\hat{s}^{2}(0)$ and $\Delta \alpha^{(3)}\left(\bar{m}_{c}\right)$. The flavor separation uncertainty is due to the imperfect knowledge of how much $s$ quarks relative to $u$ and $d$ quarks contribute to $\Delta \alpha^{(3)}\left(\bar{m}_{c}\right)$. It is given by [2],

$$
\delta \hat{s}^{2}(0) \simeq \frac{1}{20} \delta \Delta \hat{\alpha}^{(2)}\left(\bar{m}_{c}\right)= \pm 1.0 \times 10^{-5},
$$

where we used $\delta \Delta \hat{\alpha}^{(2)}\left(\bar{m}_{s}\right)= \pm 1.9 \times 10^{-4}$ from eq. (5.25).

The flavor separation assumed isospin symmetry in the form $\bar{m}_{u}=\bar{m}_{d}$. To estimate the uncertainty associated with isospin breaking, we first consider the idealized case in which $\mathrm{SU}(2)$ isospin violation was as large as $\mathrm{SU}(3)$ breaking. This would occur for $\bar{m}_{d}=\bar{m}_{s}$, so that from eq. (5.25) the $u$ quark current could at most contribute

$$
\Delta \alpha^{(1)}\left(\bar{m}_{d}\right)<14.8 \times 10^{-4} .
$$

To propagate this uncertainty to $\hat{s}^{2}(0)$ we can use [2],

$$
\delta \hat{s}^{2}(0)=-\frac{3}{40} \Delta \alpha^{(1)}\left(\bar{m}_{d}\right)>-1.1 \times 10^{-4} .
$$

A measure of the breaking of $\mathrm{SU}(2)$ relative to $\mathrm{SU}(3)$ is given by the ratio,

$$
\left|\frac{M_{K^{* \pm}}^{2}-M_{K^{* 0}}^{2}}{M_{K^{* \pm}}^{2}-M_{\rho^{0}}^{2}}\right| \approx 0.06,
$$

so that,

$$
\delta \hat{s}^{2}(0)={ }_{-7}^{+0} \times 10^{-6} .
$$

This error is asymmetric because we assume $\bar{m}_{d} \geq \bar{m}_{u}$, but it is convenient and conservative to treat it symmetrically in table 4 .

The uncertainty arising from the singlet contribution is given in eq. (4.11). The last entry in table 4 combines the truncation error from the perturbative matching conditions with the scheme conversion error shown as the second uncertainty in eq. (3.7).

\section{$7 \quad$ Results and conclusions}

Eq. (2.5) together with the $Z$ pole value of the weak mixing angle from a global fit to the SM [35], $\sin ^{2} \hat{\theta}_{W}\left(M_{Z}\right)=0.23129(5)$, can now be used to compute the weak mixing angle at zero momentum transfer,

$$
\sin ^{2} \hat{\theta}_{W}(0)=0.23868 \pm 0.00005 \pm 0.00002,
$$

where the second error is the total theoretical uncertainty from table 4 .

To facilitate the update of our results in the future, we also present a linearized formula of the form factor $\kappa(0)$,

$$
\sin ^{2} \hat{\theta}_{W}(0) \equiv \hat{\kappa}(0) \sin ^{2} \hat{\theta}_{W}\left(M_{Z}\right),
$$

in terms of variations of the input parameters, using $\Delta \hat{\alpha}_{s}\left(M_{Z}\right)$ in eq. (3.6), as well as,

$$
\tilde{\Delta} \alpha \equiv \Delta \alpha(2.0 \mathrm{GeV})-0.005871
$$




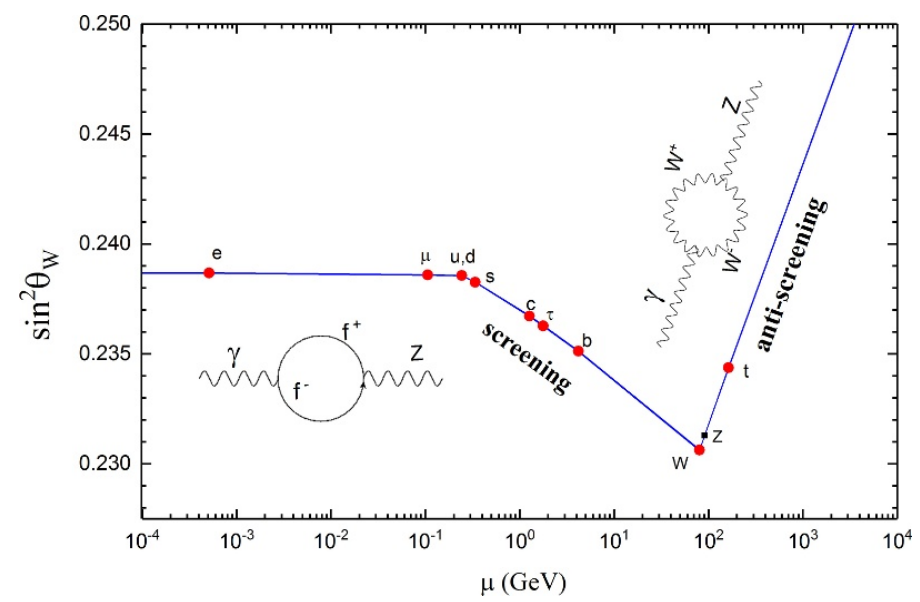

Figure 3. Scale dependence of the weak mixing angle in the $\overline{\mathrm{MS}}$ renormalization scheme. The dots indicate the scales where a particle is integrated out. The total uncertainty corresponds to the thickness of the line. The $\beta$-function of $\mathrm{SU}(2)_{L}$ changes sign at $\mu=M_{W}$, where the fermionic screening effects of the effectively Abelian gauge theory are being overcompensated by the antiscreening effects of the full non-Abelian electroweak theory.

and,

$$
\Delta \hat{m}_{c} \equiv \frac{\hat{m}_{c}\left(\hat{m}_{c}\right)}{1.272 \mathrm{GeV}}-1, \quad \Delta \hat{m}_{b} \equiv \frac{\hat{m}_{b}\left(\hat{m}_{b}\right)}{4.180 \mathrm{GeV}}-1
$$

We obtain,

$$
\hat{\kappa}(0)=1.03196 \pm 0.00006+1.14 \tilde{\Delta} \alpha+0.025 \Delta \hat{\alpha}_{s}-0.0016 \Delta \hat{m}_{c}-0.0012 \Delta \hat{m}_{b},
$$

which shows that the current experimental uncertainties of $\pm 0.45 \times 10^{-4}$ in $\Delta \alpha(2 \mathrm{GeV})$ from eq. (4.9) and of \pm 0.0016 in $\hat{\alpha}_{s}\left(M_{Z}\right)$ induce errors of $\pm 5 \times 10^{-5}$ and $\pm 4 \times 10^{-5}$ in $\hat{\kappa}(0)$, respectively. Variations of $\pm 8 \mathrm{MeV}[37]$ in $\hat{m}_{c}\left(\hat{m}_{c}\right)$ and $\pm 30 \mathrm{MeV}$ in $\hat{m}_{b}\left(\hat{m}_{b}\right)$ both imply $\mp 2 \times 10^{-6}$ in $\hat{s}(0)$ which is negligible. The resulting scale evolution of the weak mixing angle is illustrated in figure 3 .

When our result for the weak mixing angle in the Thomson limit or some other low momentum scale is used for the calculation of physical observables, there will generally be further process-dependent radiative corrections which need to be addressed. We expect this to be possible with theoretical uncertainties well below those in $\sin ^{2} \hat{\theta}_{W}(0)$ summarized in table 4 . Thus, we reduced the total theoretical uncertainty in the weak mixing angle at low energies from $7 \times 10^{-5}$ [2] to less than $2 \times 10^{-5}$ which can safely be neglected for any current or planned experiment.

In summary, we developed a new way of calculating the flavor separation which involved both $e^{+} e^{-} \rightarrow$ hadrons data and results from lattice gauge theory. We also better control now the uncertainty in the contribution of disconnected diagrams where we exploited results of ref. [23] on the anomalous magnetic moment. Furthermore, we extended various formulas to the next order in perturbation theory, reducing the perturbative uncertainty. There has also been significant progress in the evaluations of $\Delta \alpha[16,38]$ and $\Delta \hat{m}_{c}\left(\hat{m}_{c}\right)$ [37]. The theoretical uncertainty in $\sin ^{2} \hat{\theta}_{W}(0)$ is now at a negligible level. 


\section{Acknowledgments}

This work is supported by CONACyT (Mexico) project 252167-F and the German-Mexican research collaboration grant SP 778/4-1 (DFG) and 278017 (CONACyT).

Note added. When this paper was under revision, a new analysis of $\alpha\left(M_{Z}^{2}\right)$ appeared [41]. Their value,

$$
\alpha\left(M_{Z}^{2}\right)^{-1}=128.946 \pm 0.015,
$$

is even closer to our result (A.6) than the previous analysis in ref. [39]. However, there are some non-negligible differences in specific channels between refs. [16] and [41].

\section{A Calculations of $\alpha\left(M_{Z}^{2}\right)$}

Three independent groups presented recent evaluations of the hadronic contribution to the scale dependence of $\alpha$. In this appendix we briefly compare their approaches and results.

In the Adler function approach [33, 38], one uses the relations,

$$
\frac{D\left(Q^{2}\right)}{Q^{2}} \equiv 12 \pi^{2} \frac{d \Pi\left(q^{2}\right)}{d q^{2}}=-\frac{3 \pi}{\alpha} \frac{d}{d q^{2}} \Delta_{\text {had }}\left(q^{2}\right)=\int_{4 m^{2}}^{\infty} \frac{R(s)}{\left(s+Q^{2}\right)^{2}} d s,
$$

where $Q^{2}=-q^{2}$, and where the dispersion integral in the latter expression can be used to implement experimental data up to some cut-off $M_{0}$. One can then write,

$$
\begin{aligned}
\Delta_{\mathrm{had}} \alpha^{(5)}\left(M_{Z}^{2}\right)= & \Delta_{\mathrm{had}} \alpha^{(5)}\left(-M_{0}^{2}\right)_{\text {data }}+\left[\Delta_{\mathrm{had}} \alpha^{(5)}\left(-M_{Z}^{2}\right)-\Delta_{\mathrm{had}} \alpha^{(5)}\left(-M_{0}^{2}\right)\right]_{\mathrm{PQCD}} \\
& +\left[\Delta_{\mathrm{had}} \alpha^{(5)}\left(M_{Z}^{2}\right)-\Delta_{\mathrm{had}} \alpha^{(5)}\left(-M_{Z}^{2}\right)\right]_{\mathrm{PQCD}},
\end{aligned}
$$

where the last two terms are computed using the operator product expansion (OPE) of $R(s)$, i.e., including the leading non-perturbative condensate corrections. Demanding consistency with the OPE of the Adler function itself suggests that a value of $M_{0}$ as low as $2 \mathrm{GeV}$ appears to be a safe choice. Using this approach implies [38] for the on-shell definition,

$$
\alpha\left(M_{Z}^{2}\right)^{-1}=128.958 \pm 0.016
$$

The approach of ref. [39] is mostly data driven. Experimental data were used up to $11.09 \mathrm{GeV}$ (except for the interval between $2.6 \mathrm{GeV}$ and $3.73 \mathrm{GeV}$ ) and PQCD beyond that. The dispersion relation (3.1) then implied,

$$
\alpha\left(M_{Z}^{2}\right)^{-1}=128.944 \pm 0.019 .
$$

Similarly, ref. [16] uses data up to only $5 \mathrm{GeV}$ (except for the interval between $1.8 \mathrm{GeV}$ and $3.7 \mathrm{GeV}$ ), with the result,

$$
\alpha\left(M_{Z}^{2}\right)^{-1}=128.947 \pm 0.012
$$

Here, we rely on the data handling of this work as it includes much more recent data than ref. [39]. Moreover, the breakdown of individual channels and energy ranges is more explicit compared to ref. [38]. 
Finally, changing our own result, with $\hat{\alpha}\left(M_{Z}^{2}\right)^{-1}=127.959 \pm 0.010$, based on the direct application of the renormalization group and matching equations and including $\tau$ decay data, from the $\overline{\mathrm{MS}}$ scheme to the on-shell scheme including the top quark contribution, we find,

$$
\alpha\left(M_{Z}^{2}\right)^{-1}=128.946 \pm 0.010
$$

The numerical difference of our result to ref. [16] arises mostly from the different ${ }^{3}$ value of $\alpha_{s}$ and our treatment of the charm quark contribution [37]. Thus, in view of the rather different approaches and differences in data sets, all numerical results are in good agreement with each other.

\section{B Calculation of $\Delta_{\text {disc }} \hat{\alpha}$}

In the on-shell scheme one has [40],

$$
\Delta_{\mathrm{disc}} \alpha(q)=4 \pi \alpha \operatorname{Re}\left[\Pi\left(q^{2}\right)-\Pi(0)\right]_{\mathrm{disc}},
$$

where,

$$
\left[\Pi\left(q^{2}\right)-\Pi(0)\right]_{\mathrm{disc}}=\sum_{t=0}^{T}\left[\frac{\cos (q t)-1}{q^{2}}+\frac{t^{2}}{2}\right] C(t) .
$$

$C(t)$ has been computed [23] in units set by the lattice cut-off scale $a^{-1}=1.73 \mathrm{GeV}$. To obtain eq. (4.10), we plotted $\Delta_{\text {disc }} \alpha(q)$ as a function of $T$ and observe a plateau near $T=20$, which closely mirrors the result for the case of $a_{\mu}$. The value of the plateau is interpreted as the physical value [23]. As an independent check we compute the ratio $\rho\left(a_{\mu}\right)$ of the disconnected contribution to the anomalous magnetic moment $[23], a_{\mu}^{\text {disc }}=-9.6 \times$ $10^{-10}$, to the total hadronic contribution [16] for energies up to $1.8 \mathrm{GeV}$, obtaining $\rho\left(a_{\mu}\right)=$ -0.015. The integration kernel of $a_{\mu}$ enhances contributions from low $q^{2}$ momenta, and recalling that $Q_{u}+Q_{d}+Q_{s}=0$, the disconnected piece also predominantly arises from such momenta. On the other hand, the integration kernel for $\Delta \alpha$ has greater support at higher scales compared to $a_{\mu}$, so that $\rho\left(a_{\mu}\right)$ should imply an upper bound on the disconnected contribution to $\Delta \alpha$. Numerically,

$$
\left|\Delta_{\text {disc }} \alpha(1.8 \mathrm{GeV})\right|<\left|\rho\left(a_{\mu}\right) \times \Delta_{\text {had }} \alpha(1.8 \mathrm{GeV})\right|=8.3 \times 10^{-5}
$$

where $\Delta_{\text {had }} \alpha(1.8 \mathrm{GeV})=55.26 \times 10^{-4}$. This confirms the finding in eq. (4.10) that $\Delta_{\text {disc }} \alpha$ is very small.

Open Access. This article is distributed under the terms of the Creative Commons Attribution License (CC-BY 4.0), which permits any use, distribution and reproduction in any medium, provided the original author(s) and source are credited.

\footnotetext{
${ }^{3}$ The three groups use slightly different values for $\alpha_{s}$, but this amounts to difference below the level of 0.004 in $\alpha\left(M_{Z}\right)^{-1}$.
} 


\section{References}

[1] A. Czarnecki and W.J. Marciano, Polarized Moller scattering asymmetries, Int. J. Mod. Phys. A 15 (2000) 2365 [hep-ph/0003049] [INSPIRE].

[2] J. Erler and M.J. Ramsey-Musolf, The weak mixing angle at low energies, Phys. Rev. D 72 (2005) 073003 [hep-ph/0409169] [INSPIRE].

[3] Qweak collaboration, D. Androic et al., First determination of the weak charge of the proton, Phys. Rev. Lett. 111 (2013) 141803 [arXiv:1307.5275] [INSPIRE].

[4] D. Becker et al., The P2 experiment - a future high-precision measurement of the electroweak mixing angle at low momentum transfer, arXiv:1802.04759 [INSPIRE].

[5] MOLLER collaboration, J. Benesch et al., The MOLLER experiment: an ultra-precise measurement of the weak mixing angle using Møller scattering, Jefferson Lab document JLAB-PHY-14-1986, U.S.A., (2014) [arXiv:1411.4088] [INSPIRE].

[6] SLAC E158 collaboration, P.L. Anthony et al., Precision measurement of the weak mixing angle in Moller scattering, Phys. Rev. Lett. 95 (2005) 081601 [hep-ex/0504049] [INSPIRE].

[7] PVDIS collaboration, D. Wang et al., Measurement of parity violation in electron-quark scattering, Nature 506 (2014) 67 [INSPIRE].

[8] P.A. Souder, Parity violation in deep inelastic scattering with the SoLID Spectrometer at JLab, Int. J. Mod. Phys. Conf. Ser. 40 (2016) 1660077 [inSPIRE].

[9] NuTEV collaboration, G.P. Zeller et al., A precise determination of electroweak parameters in neutrino nucleon scattering, Phys. Rev. Lett. 88 (2002) 091802 [Erratum ibid. 90 (2003) 239902] [hep-ex/0110059] [INSPIRE].

[10] B.C. Canas, E.A. Garces, O.G. Miranda, M. Tortola and J.W.F. Valle, The weak mixing angle from low energy neutrino measurements: a global update, Phys. Lett. B 761 (2016) 450 [arXiv: 1608.02671] [INSPIRE].

[11] C.S. Wood et al., Measurement of parity nonconservation and an anapole moment in cesium, Science 275 (1997) 1759 [INSPIRE].

[12] L. Willmann et al., Trapped radioactive isotopes for fundamental symmetry investigations. The TRI $\mu$ P facility, Hyperfine Interact. 211 (2012) 39.

[13] K.S. Kumar, S. Mantry, W.J. Marciano and P.A. Souder, Low energy measurements of the weak mixing angle, Ann. Rev. Nucl. Part. Sci. 63 (2013) 237 [arXiv:1302.6263] [INSPIRE].

[14] J. Erler and S. Su, The weak neutral current, Prog. Part. Nucl. Phys. 71 (2013) 119 [arXiv: 1303.5522] [INSPIRE].

[15] J. Erler, C.J. Horowitz, S. Mantry and P.A. Souder, Weak polarized electron scattering, Ann. Rev. Nucl. Part. Sci. 64 (2014) 269 [arXiv:1401.6199] [InSPIRE].

[16] M. Davier, A. Höcker, B. Malaescu and Z. Zhang, Reevaluation of the hadronic vacuum polarisation contributions to the Standard Model predictions of the muon $g-2$ and $\alpha\left(m_{Z}^{2}\right)$ using newest hadronic cross-section data, Eur. Phys. J. C 77 (2017) 827 [arXiv: 1706.09436] [INSPIRE].

[17] F. Jegerlehner and R. Szafron, $\rho^{0}-\gamma$ mixing in the neutral channel pion form factor $F_{\pi}^{e}$ and its role in comparing $e^{+} e^{-}$with $\tau$ spectral functions, Eur. Phys. J. C 71 (2011) 1632 [arXiv:1101.2872] [INSPIRE]. 
[18] RBC/UKQCD collaboration, T. Blum et al., Lattice calculation of the leading strange quark-connected contribution to the muon g-2, JHEP 04 (2016) 063 [Erratum ibid. 05 (2017) 034] [arXiv: 1602.01767] [INSPIRE].

[19] HPQCD collaboration, B. Chakraborty et al., Strange and charm quark contributions to the anomalous magnetic moment of the muon, Phys. Rev. D 89 (2014) 114501 [arXiv:1403.1778] [INSPIRE].

[20] S. Okubo, $\varphi$ meson and unitary symmetry model, Phys. Lett. 5 (1963) 165 [INSPIRE].

[21] G. Zweig, An SU(3) model for strong interaction symmetry and its breaking II, CERN-TH-412, CERN, Geneva Switzerland, (1964).

[22] J. Iizuka, Systematics and phenomenology of meson family, Prog. Theor. Phys. Suppl. 37 (1966) 21 [INSPIRE].

[23] RBC/UKQCD collaboration, T. Blum et al., Calculation of the hadronic vacuum polarization disconnected contribution to the muon anomalous magnetic moment, Phys. Rev. Lett. 116 (2016) 232002 [arXiv:1512.09054] [INSPIRE].

[24] J. Erler, Calculation of the QED coupling $\alpha\left(M_{Z}\right)$ in the modified minimal subtraction scheme, Phys. Rev. D 59 (1999) 054008 [hep-ph/9803453] [INSPIRE].

[25] P.A. Baikov, K.G. Chetyrkin, J.H. Kühn and J. Rittinger, Vector correlator in massless $Q C D$ at order $O\left(\alpha_{s}^{4}\right)$ and the QED $\beta$-function at five loop, JHEP 07 (2012) 017 [arXiv: 1206.1284] [INSPIRE].

[26] T. van Ritbergen, J.A.M. Vermaseren and S.A. Larin, The four loop $\beta$-function in quantum chromodynamics, Phys. Lett. B 400 (1997) 379 [hep-ph/9701390] [INSPIRE].

[27] M. Czakon, The four-loop QCD $\beta$-function and anomalous dimensions, Nucl. Phys. B 710 (2005) 485 [hep-ph/0411261] [INSPIRE].

[28] L.J. Hall, Grand unification of effective gauge theories, Nucl. Phys. B 178 (1981) 75 [INSPIRE].

[29] K.G. Chetyrkin, B.A. Kniehl and M. Steinhauser, Decoupling relations to $O\left(\alpha_{s}^{3}\right)$ and their connection to low-energy theorems, Nucl. Phys. B 510 (1998) 61 [hep-ph/9708255] [INSPIRE].

[30] K.G. Chetyrkin, J.H. Kühn and C. Sturm, Four-loop moments of the heavy quark vacuum polarization function in perturbative QCD, Eur. Phys. J. C 48 (2006) 107 [hep-ph/0604234] [INSPIRE].

[31] B.A. Kniehl and A.V. Kotikov, Heavy-quark QCD vacuum polarisation function: analytical results at four loops, Phys. Lett. B 642 (2006) 68 [hep-ph/0607201] [INSPIRE].

[32] R. Alemany, M. Davier and A. Höcker, Improved determination of the hadronic contribution to the muon $(g-2)$ and to $\alpha\left(M_{Z}^{2}\right)$ using new data from hadronic $\tau$ decays, Eur. Phys. J. C 2 (1998) 123 [hep-ph/9703220] [INSPIRE].

[33] S. Eidelman, F. Jegerlehner, A.L. Kataev and O. Veretin, Testing nonperturbative strong interaction effects via the Adler function, Phys. Lett. B 454 (1999) 369 [hep-ph/9812521] [INSPIRE].

[34] J. Erler and R. Ferro-Hernández, Calculation of the QED coupling $\alpha\left(M_{Z}^{2}\right)$ in the $\overline{\mathrm{MS}}$ scheme: an update, in preparation. 
[35] Particle Data Group collaboration, C. Patrignani et al., Review of particle physics, Chin. Phys. C 40 (2016) 100001 [INSPIRE].

[36] J. Erler and M.-X. Luo, Hadronic loop corrections to the muon anomalous magnetic moment, Phys. Rev. Lett. 87 (2001) 071804 [hep-ph/0101010] [INSPIRE].

[37] J. Erler, P. Masjuan and H. Spiesberger, Charm quark mass with calibrated uncertainty, Eur. Phys. J. C 77 (2017) 99 [arXiv: 1610.08531] [InSPIRE].

[38] F. Jegerlehner, Variations on photon vacuum polarization, arXiv:1711.06089 [INSPIRE].

[39] K. Hagiwara, R. Liao, A.D. Martin, D. Nomura and T. Teubner, $(g-2)_{\mu}$ and $\alpha\left(M_{Z}^{2}\right)$ re-evaluated using new precise data, J. Phys. G 38 (2011) 085003 [arXiv:1105.3149] [INSPIRE].

[40] D. Bernecker and H.B. Meyer, Vector correlators in lattice QCD: methods and applications, Eur. Phys. J. A 47 (2011) 148 [arXiv:1107.4388] [inSPIRE].

[41] A. Keshavarzi, D. Nomura and T. Teubner, The muon $g-2$ and $\alpha\left(M_{Z}^{2}\right)$ : a new data-based analysis, arXiv:1802.02995 [INSPIRE]. 